\title{
Recombination Coefficient of Heavy Elements Using Photoionization Modeling
}

\author{
Belay Sitotaw Goshu ${ }^{1,2}$ \\ ${ }^{1}$ Department of Physics, Dire-Dawa University, Dire-Dawa, Ethiopia \\ ${ }^{2}$ Department of Mathematics, Astronomy and Computing Science, Unisa, South Africa
}

Email address:

belaysitotaw@gmail.com

\section{To cite this article:}

Belay Sitotaw Goshu. Recombination Coefficient of Heavy Elements Using Photoionization Modeling. American Journal of Astronomy and Astrophysics. Vol. 5, No. 4, 2017, pp. 42-49. doi: 10.11648/j.ajaa.20170504.11

Received: June 10, 2017; Accepted: June 23, 2017; Published: September 28, 2017

\begin{abstract}
The main purpose of this work is to calculate the radiative, dielectronic and total recombination of nonhydrogenic elements. We applied the photoionization codes to investigate the recombination coefficients. We have calculated radiative and dielectronic recombination coefficients of some elements at different temperatures and ionization stages. The results are obtained at low temperatures between 5,000 and 20,000 K and it shows that the dielectronic coefficients are not negligible relative to radiative recombination by assuming the solar abundances of $\mathrm{H}, \mathrm{He}, \mathrm{C}, \mathrm{N}, \mathrm{O}, \mathrm{Ne}$ and $\mathrm{S}$. We determined the total recombination coefficients of each element and compared them with previous works. Our calculations the radiative dielectric and total recombination coefficients of oxygen, nitrogen, carbon, neon and sulphur ions at a given temperature agree with the previous works.
\end{abstract}

Keywords: Photoionization, Radiative, Dielectronic Recombination Coefficients

\section{Introduction}

Recombination of electrons and ions during scattering processes, which commonly occurs in many gaseous nebulae and plasma environments, is a fundamental process that has a strong impact on many physical systems. Recombination lines produced by such physical process can be used as a diagnostic tool to determine elemental abundances and electron temperatures in $\mathrm{H}$ II regions and planetary nebulae.

Recombination rates are needed in studies of the ionization state of plasma departing from thermodynamic equilibrium. Recombination followed by cascades is important in populating bound atomic levels of energy much larger than the thermal kinetic energy of the gas. There are different types of recombination [1].

The total effective, radiative and dielectronic recombination rates of ions are needed in studies of the ionization state of a plasma in thermodynamic equilibrium. Recombination followed by cascades is important in populating bound atomic levels of energy much larger than the thermal kinetic energy of the gas. This calculation is based on the approximation proposed by [2].
Tabulations of fits to radiative recombination coefficients have been done by [3, 4, 5, and 6] while [7] improve some of the results at two temperatures cited in [1]. But in this work, we used cloudy computational techniques to tackle the problems.

Cloudy is an impressive code offering a vast amount of possibilities to model a wide variety of objects. The main physics and basics of a numerical framework of the code are explained in "Hazy: a brief introduction to the code" [8]. The model presented here use version $\mathrm{C} 10.00$ of the code developed by [8]. It contains most of the atomic processes that are thought to be important in the temperature range $\left(\sim 10^{4}-10^{8} K\right)$. Moreover, cloudy needs to specify the shape and the intensity of radiation field and the gas density, its chemical composition and the geometry of the cloud.

The main goal of this work is to calculate the radiative, dielectronic and the total recombination coefficients of nonhydrogenic ions of the elements which are most abundant elements like $\mathrm{C}, \mathrm{N}, \mathrm{O}, \mathrm{Ne}$ and $\mathrm{S}$. We assume low-density plasma (optically thin) of nebulae heated by energetic photons in the interstellar medium and nebulae; recombination mainly proceeds from the ground state of ions.

The organization of this paper is as follows, in section II, 
we describe the photoionization equilibrium of ions and atoms, in section III, both radiative and dielectronic recombination are presented while in section III, we discuss the total recombination, dielectronic and radiative recombination coefficients of some ions, in section IV we presents the model parameters. In section $\mathrm{V}$ we present the results and compared with the previous work done by different scholars should be and compared them with the previous works and conclusion is presented in the last section.

\section{Photoionization Equilibrium}

The Universe contains copious sources of energetic photons most often in the form of hot stars, and much of the material of the Universe exists as a photoionized gas. Photoionized gas produces the visible emission from emission nebulae, planetary nebulae, nova shells, starburst galaxies and probably active galactic nuclei [9]. Emission nebulae are extended regions of luminosity in the sky. They arise from the absorption of stellar radiation by the gas surrounding one or hotter stars. The gas is ionized by the photons and excited and heated by the electrons released in the photoionizing events. A succession of ionization zones is created in which highly ionized regions give way to less ionized gas with increasing distance from the central star as the photon flux is diminished by geometrical dilution and by absorption. The outer edge of a nebula is a front of ionization pushing out into the neutral interstellar gas.

In ionization equilibrium, photoionization is balanced by recombination. Thermal equilibrium is achieved when photoionization and Compton heating are balanced by collisional cooling, recombination, line excitation, bremsstrahlung and Compton cooling. The conditions under which these equilibriums are achieved depending on the shape of the continuum, the metalicity of the gas, the density, the column density and the ratio of the ionizing photon flux to the gas density [10]. For a cloud of hydrogen-containing an ionizing source, the equilibrium between photoionization and recombination of protons and electrons in nebulae can be written [9].

The ionization equilibrium at each point in the nebulae is fixed by the balance between photoionization and recombination of electrons with ions. Since hydrogen is the most abundant element, we can get a first idealized approximation to the structure of nebulae by considering a cloud surrounding a single hot star. For a cloud of hydrogencontaining an ionizing source, the equilibrium between photoionization and recombination of protons and electrons in nebulae can be formed [9],

$$
\int_{v_{o}}^{\infty} \frac{L_{v}}{h v} d v=4 \pi \int_{0}^{r_{i o n}} n_{p} n_{e} \alpha_{A, B} r^{2} d r
$$

where $L_{v}$ is the specific luminosity at a frequency $v$ and $h$ is the Planck's constant, giving the numbers of ionizing photons per seconds, $v_{o}$ is the threshold frequency of ions, $r_{i o n}$ is the extent of the ionization, $n_{p}$ and $n_{e}$ are the photon and electron densities, and the $\alpha_{A, B}$ the radiative recombination rate coefficients of elements. We now parameterized the right- hand side of the photoionization equilibrium Eqn. 1. Since we are concerned with the ionization of the neutral gas and its subsequent recombination, $\mathrm{n}=\mathrm{n}_{\mathrm{e}}=\mathrm{n}_{\mathrm{p}}$.

The photoionization of an atom leads to an absorption of that photon. That means that photoionization causes opacity depends on energy. The opacity of hydrogen-like ions with atomic number $\mathrm{Z}$ is given by

$$
a_{v}=\propto a_{o} \frac{1}{Z^{2}}\left(\frac{v_{o}}{v}\right)^{4} \frac{e^{4-\frac{4 \tan ^{-1} \epsilon}{\epsilon}}}{1-e^{\frac{-2 \pi}{\epsilon}}}
$$

Where $\epsilon=\sqrt{\frac{v}{v_{o}}-1}$ for $v>v_{o}$

Eqn. 2 shows that the photoionization cross section peaks at the threshold frequency $v_{o}$ and drops quickly toward high energy. This is due to high hydrogen and helium ratios, photons with energies much higher than $13.6 \mathrm{eV}$ penetrate further into gas before they get absorbed than photons with energies close to this threshold.

The integrated volume of Stromgren sphere for the analytical purpose can be calculated from Eqn. 1. The total number of ionizing photons of the source is exactly balanced by the total number of recombination excited levels. The total number of ionizing absorbs all ionizing photons reformulated

$$
Q(H)=\frac{4}{3} \pi n^{2} \alpha_{A}(H I, T) R_{S}{ }^{3}
$$

Where is $R_{S}$ Stromgren sphere of the radius and is $\alpha_{B}$ the recombination coefficients. Therefore, we may write Eqn. 1 as

$$
\int_{v_{o}}^{\infty} \frac{L_{v}}{h v} d v=\frac{4 \pi}{3} n^{2} \alpha_{A}(H I, T) R_{S}{ }^{3}
$$

In a spherically symmetric model, the photoionized nebulae surrounded by a central point source is emitting ionizing radiation. This geometry represents HII regions when they are emitting gas surrounded the central ionizing stars. The radiation field emitted is assumed to be outward only. That is, any radiation passes through the ionized region is absorbed. The radius of the sphere whose central nebula contains a mixture of hydrogen and helium is given by

$$
R_{S}=\left|\frac{3}{4 \pi} \frac{Q}{\alpha_{A}(H I, T)}\right|^{1 / 3} N_{H I}^{-2 / 3}
$$

The radius derived by Eqn. 3 is usually referred to as the radius of Stromgren radius after B. Stromgren who first derived this equation in 1939. The sphere of ionized hydrogen is called a Stromgren sphere.

\section{Total Recombination Coefficients}

Total radiative recombination is the sum of direct recombination onto all atomic levels. Direct recombination coefficients are computed from photoionization cross section by means of Miline relation $[1,11]$. The total recombination rates $\alpha\left(X^{i}, T\right)$, calculated for all $\mathrm{C}, \mathrm{N}, \mathrm{O}, \mathrm{Ne}$ and $\mathrm{S}$ ions in 
this work, are calculated using photoionization as in radiatively ionized sources to obtain ionization equilibrium corresponding to plasmas ionized by electron impact ionization [12]. The ionization balance equation described in Eqn. 1 can be rewritten as in photoionization equilibrium as

$$
\int_{v_{o}}^{\infty} \frac{4 \pi J_{v}}{h v} N(Z-1) \sigma_{p i}(v, X(z)) d v=N_{e} N_{p} \alpha_{R}(z, T)
$$

Where $\sigma_{p i}$ the photoionization cross section evaluated at photon frequency $v$ and convoluted by an isotropic radiation density $J_{v}$ of the source; $N_{e}$ is the density of the free electrons. These ionization fractions can be evaluated using the present total recombination rate coefficients and photoionization cross sections shown in [12].

The total recombination coefficient rate is the total sum of both radiative and dielectronic recombination, which is described as (DR + RR). Where $R R$ is the radiative recombination of an electron and ion may take place through a background continuum and DR is the dielectronic recombination coefficients through a resonant recombination process involving double excited states. The total recombination coefficients for complex ion

$$
\alpha_{T}\left(X^{+i}, T\right)=\alpha_{R}\left(X^{+i} . T\right)+\alpha_{d}\left(X^{i+}, T\right)+\sum_{n>n_{g}}^{N} \alpha_{n r}\left(X^{+i}, T\right)
$$

Where is $\alpha_{T}\left(X^{+i}, T\right)$ the total, $\alpha_{R}\left(X^{+i} . T\right)$ is radiative, $\alpha_{n r}\left(X^{+i}, T\right)$ summation extends over all excited states and is $\alpha_{d}\left(X^{i+}, T\right)$ dielectronic recombination coefficients of ions with the total number of elements with the atomic number. These partial recombination coefficients (to level $n$ ) follow from the Miline relation of detailed balance [13] and mentioned in [14].

The radiative part represents $\alpha_{R}\left(X^{+i} . T\right)$ simple bound free recaptures. Just in $\mathrm{H}$ and $\mathrm{He}$ captures to any level are followed by transitions, leading ultimately to the ground level. The radiative recombination coefficient is the sum of all levels and is dominated by the excited levels, which to a good approximation are hydrogen and helium like.

\subsection{Radiative Recombination Coefficients}

Radiative recombination is the process of capture of an electron by an ion with the excess energy being radiated away in a photon. In most cases electron is captured into a state of large principal quantum number and high angular momentum state, so that the recombined, excited, ion radiates several photons in a radiative cascade back down to the ground state [15].

$$
X^{i}+e^{-} \rightarrow X^{i-1}+h v
$$

Where is $X^{i}$ ion of effective charge, e is a charge of the electron, $h$ is the Planck's constant, $v$ is the frequency and is $X^{i-1}$ an excited bound state of the recombined ion with the effective charge $i-1$.

Most of the energy input comes from photoionization. Every photon is absorbed by a hydrogen atom generates a free electron energy. The rate of radiative rises as the free electron temperature falls and hence it tends to be the dominant process at low temperature. The energy of the system is conserved, so the kinetic energy of the free electron and the energy of emitted photon are related by

$$
h v=\chi_{n}+\frac{1}{2} m_{e} v^{2}
$$

Where $\chi_{n}$ is the ionization potential of the level $\mathrm{n}$ of the atom, me is the mass of the electron and $\mathrm{v}$ is the free electron speed. The mean initial energy of electrons depends on the shape of the ionizing spectrum.

The radiative recombination rate to a level $\mathrm{n}$ per unit volume is dependent on the cross-section for radiative recombination $\sigma_{n}$, of ions and electron described can be written as should be of ions and electrons described by $v=\sqrt{\frac{2 \varepsilon}{m}}$ and $f(v ; T)$ is the velocity distribution function [16]. The radiative recombination rate coefficient $\alpha_{n}^{R}$ is found by averaging the cross-section over the velocity distribution function of $f(v)$ the free electrons. It also depends on the number densities of the free electrons and the ions, $n_{e}$, and $n_{i}$ respectively, and is given by

$$
n_{e} n_{i} \int_{v_{o}}^{\infty} \sigma_{n}(v) f(v) d v=n_{e} n_{i} \alpha_{n}^{R}
$$

Collisions amongst the free electrons set up a Maxwellian distribution for these particles on time scales that are much shorter than other atomic processes. Therefore, the Maxwellian distribution is given by

$$
f(v) d v=4 \pi v^{2}\left(\frac{m_{e}}{2 \pi K_{B} T_{e}}\right)^{\frac{3}{2}} e^{m_{e} v^{2} /\left(3 K_{B} T_{e}\right)} d v
$$

The recombination coefficient at a particular quantum level $n$ is defined as

$$
\alpha_{n}=<v \sigma_{n}(v)>\int v f(v) \sigma_{n}\left(v, X^{i}\right) d v
$$

and acquires knowledge of the velocity distribution of the electrons. The cross section for radiative capture $\sigma_{n}\left(v, X^{i}\right)$, can be found from the standard relation of Miline [9], involving the equilibrium condition for the processes described by the interaction of ions and electron described can be written as

$$
\sigma_{n}\left(v, X^{i}\right)=\frac{\omega_{i}}{\omega_{e} \omega_{+}}\left(\frac{2 h v}{m_{e} c v}\right)^{2} a\left(v, X^{i}\right)
$$

where $v$ and $m_{e}$ are respectively electron velocity and mass, $\omega_{+}$the statistical weight of the recombining ion, $\omega_{i}$ and $\omega_{e}$ is the statistical weight of element and free electron respectively and is $a\left(v, X^{i}\right)$ the cross-section of the element.

For a given ion of an element $X^{+i}$ the radiative recombination rate to level $\mathrm{n}$ per unit time and volume is given in terms of the number densities of the more highly ionized ion $n\left(X^{i}\right)$ and of the electrons, ne, and in terms of the electron capture cross-section to level $\mathrm{n}$ of the ion $X^{+1}$ at given electron velocity $\mathrm{v}$; the total effective recombination rate to all states can then be written as the sum of the recombination rate to reach state [17].

$$
\alpha_{\text {rad }}^{X, i}\left(T_{e}\right)=\sum_{n} \alpha\left(n, T_{e}\right)
$$


For hydrogenic ions, the radiative recombination rates can be calculated by [2]. This can be approximated by a simple power law as described in Eqn. 15.

$$
\alpha_{R}^{H}\left(T_{e}\right)=4.18 \times 10^{-13}\left(\frac{T}{10^{4}}\right)^{-0.72} \mathrm{~cm}^{-3} \mathrm{~S}^{-1}
$$

Which is applicable to the rate 5000 to $20000 \mathrm{~K}[15,6,17]$ for the radiative recombination rates, the calculations of [19], were used for some abundant astrophysical elements $(\mathrm{S}$, and $\mathrm{N})$ ? For non-hydrogenic atoms, the usual way to compute the complete radiative recombination rate is to evaluate the recombination rate to the ground state using the Miline relation, and then to compute the recombination rate to the excited using the hydrogenic approximation. The result is a simple power law with temperature as described in Eqn. 15
$[15,6]$.

$$
\alpha_{R}=A_{\text {rad }}\left(\frac{T}{10^{4} K}\right)^{-\eta} c m^{-3} S^{-1}
$$

where Arad and $\eta$ are derived by [6] and have different values at different temperatures.

The radiative recombination of hydrogen of the resulting model using cloudy and the power law was given by [2] using Eqn. 15 is shown in Figure 1. We have seen from the results that the radiative recombination has the same patterns between the temperatures of 5000 and $20000 \mathrm{~K}$, typically for photoionized nebulae of solar composition [34]. It decays exponentially with the temperature.

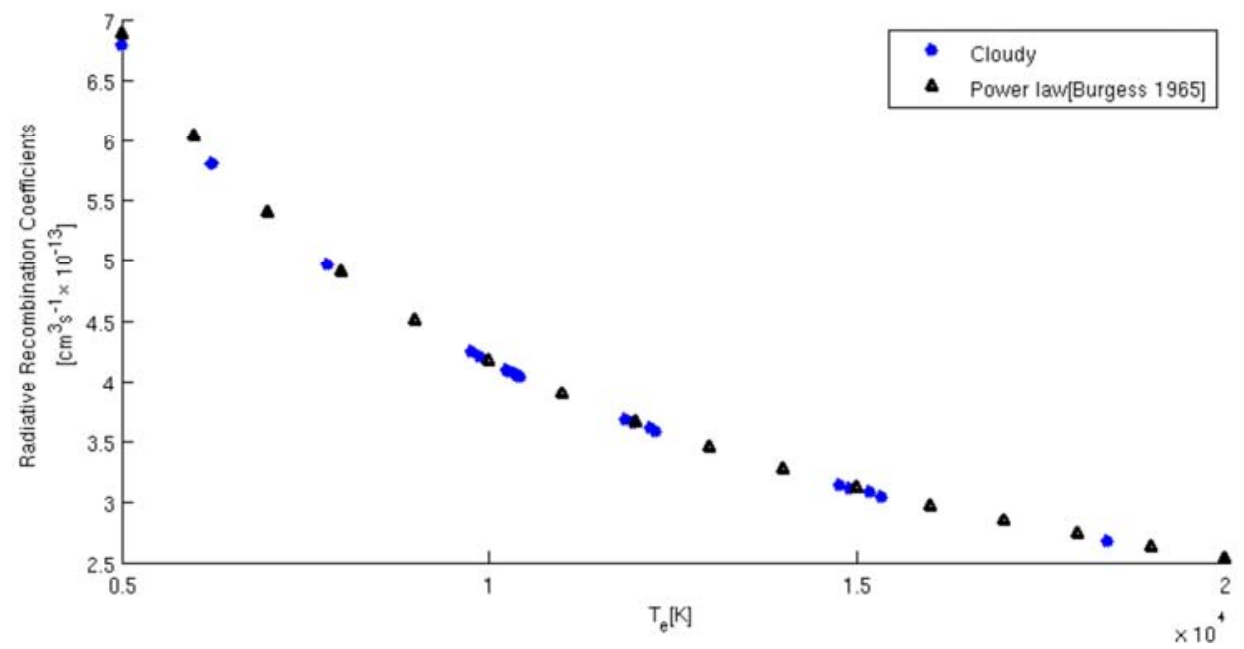

Figure 1. Radiative recombination coefficients of hydrogen using CLOUDY and power law equation.
For non-hydrogenic atoms, the usual way to compute the radiative recombination rate or to evaluate the recombination rate to the ground state is to apply the Milne relation [9], to compute the recombination rate to the excited state we can apply the hydrogenic approximation. The result is then a simple power law with temperature given by $[12,20]$

$$
\alpha_{R}=A_{\text {rad }}\left(\frac{T}{10^{4}}\right)^{-\eta} \mathrm{cm}^{-3} \mathrm{~s}^{-1}
$$

where $A_{\text {rad }}$ and $\eta$ are given by [20], which is not constant but varies from atom to atoms and ions.

\subsection{Dielectronic Recombination}

Dielectronic recombination (DR) was first investigated by $[21,22]$ as the dominant recombination channel in the solar corona [23]. After twenty years [24] investigates dielectronic recombination coefficients at low temperature. It processed to be significant at temperature interest of $T_{e} \leq 10^{4} \mathrm{~K}$ [19]. It is a two-stage process and is not exact inverse of autoionization. In the first stage, an electron in the continuum recombines with an ionic bound state. Extra energy is accepted by an electron in the bound state of the ion, which is excited to a higher bound state. Doubly autoionization or through radiative decay of one of the bound levels are the second stage. Dielectronic recombination only when radiative decay follows the recombination since there is a change in the state of ion.

The inverse of DR is photoionization [23]. It is the dominant electron-ion recombination process in many astrophysical and laboratory plasma. It plays an important role in determining both the level populations and the ionization balance of both high and low temperature nonlocal thermodynamic equilibrium (LTE) plasma over a wide range of electron densities [26]. In this process, a free electron can collisionally excite an ion and be simultaneously captured into an auto-ionizing state of the recombined system. It occurs if the resulting doubly-excited state subsequently decays radiatively below the first ionization threshold [27].

Dielectronic recombination is indirect capture and recombination through auto-ionizing states [12]. It involves forming doubly excited states which radiatively stabilize below the autoionization threshold. The recent work shows there are triply excited states for elements like Be ions. The recombination of an electron to an $n l(n)$ is the principal quantum number and 1 orbital angular momentum of an atom) sate and simultaneous excitation of the $2 \mathrm{~s}^{2}$ to $2 \mathrm{p}^{2}$. This resonance lies close to the threshold which can contribute significantly to the low energy region of the spectrum [26]. 
But in this work, we focus on free electron which is transferred to a bound state formed by an excitation of the core and a simultaneous attachment of the incident electrons. There is two-step process involves dielectronic capture as the first step with a subsequent stabilization of the lowered charge state by radiative decay to a state below the ionization limit. The second step competes with auto-ionization which would transfer the ion back to its initial state charge with the net effect being resonant elastic or inelastic scattering. The reaction is given by

$$
X^{i} \longrightarrow X^{(i-1) * *} \rightarrow h v+X^{+(i-1)}
$$

where the intermediate state (indicated by double asterisks) is double excited state of the (e+ ion) system which introduces a resonance. This can be followed either by auto-ionization to the continuum or by stabilization through a radiative decay of the captured electron. The cross sections for radiative recombination are obtained by summing the cross sections for capture into the ground and excited states of the recombining system. Because of the contribution from highly excited states which are nearly hydrogenic, the rate coefficients are similar for different ions of the same excess nuclear charge. They vary slowly with temperature [28].

The RR is usually regarded to be important at high temperatures, whereas the DR, which requires electrons energetic enough to excite the ion, comes in at higher temperatures. However, even at low temperatures, there may occur significant recombination of electrons through lowlying resonance near the threshold, where at these temperatures the free electrons can enter only these states $[29,30]$. As temperatures rise, higher auto-ionizing levels can be reached for each ion and the process becomes is complicated. Therefore, the dielectronic recombination and auto-ionization will occur rapidly.

In general, we may distinguish three types of dielectronic recombination mechanisms with the important of temperature.

(a) High-temperature dielectronic recombination which occurs through series of auto-ionizing states, in which the radiative stabilization decay within the ion.

(b) Low-temperature dielectronic recombination which operates near threshold resonance with radiative stabilization usually through decay of the outer captured the electron, which may operate at thousands to tens thousands of Kelvin.

(c) Fine structure dielectronic recombination which is due to series resonance converging on the fine structure levels on the round term at very low temperatures, which may operate at tens or hundreds of Kelvin.

The dielectronic recombination of the $\alpha_{d i}\left(X_{i}\right)$ is computed with the general expression given by [2]. This approximation holds for most ions at higher temperature $T_{e}>10^{5} \mathrm{~K}$. A simple expression can be formulated for $\alpha_{d i}(\mathrm{Te})$, given by [20], which involves a summation overall permitted transitions from the ground state of the ion. The general expression of dielectronic given by

$$
\alpha_{d i}=A_{d i} T_{e}^{-3 / 2} \exp \left(-\frac{T_{0}}{T}\right)\left[1+B_{d i} \exp \left(-\frac{T_{1}}{T e}\right)\right]
$$

Empirical values of $A_{d i}, T_{0}$, and $T_{1}$ for all ions of chemical elements from $\mathrm{C}$ to $\mathrm{S}$ were determined by a least-squares procedures from rates of [31] is shown in Table 1.

Table 1. Fits to Ratio Coefficients adopted from [31].

\begin{tabular}{lllll}
\hline Ion & Adi & Bdi & To & T1 \\
\hline C1 & $2.54 \mathrm{E}-3$ & $4.42 \mathrm{E}-2$ & $1.57 \mathrm{E} 5$ & $3.74 \mathrm{E} 5$ \\
$\mathrm{C} 2$ & $6.15 \mathrm{E}-3$ & $5.88 \mathrm{E}-3$ & $1.41 \mathrm{E} 5$ & $1.41 \mathrm{E} 5$ \\
$\mathrm{~N} 1$ & $2.98 \mathrm{E}-3$ & 0 & $2.2 \mathrm{E} 5$ & 0 \\
$\mathrm{~N} 2$ & $7.41 \mathrm{E}-3$ & $7.64 \mathrm{E}-2$ & $2.01 \mathrm{E} 5$ & $7.37 \mathrm{E} 4$ \\
$\mathrm{O} 1$ & $1.11 \mathrm{E}-3$ & $9.25 \mathrm{E}-2$ & $1.75 \mathrm{E} 5$ & $1.45 \mathrm{E} 5$ \\
$\mathrm{O} 2$ & $5.07 \mathrm{E}-3$ & 0.181 & $1.98 \mathrm{E} 5$ & $3.35 \mathrm{E} 5$ \\
$\mathrm{Ne} 1$ & $9.77 \mathrm{E}-4$ & $7.30 \mathrm{E}-2$ & $3.11 \mathrm{E} 5$ & $2.06 \mathrm{E} 5$ \\
$\mathrm{Ne} 2$ & $2.65 \mathrm{E}-3$ & 0.242 & $2.84 \mathrm{E} 5$ & $3.07 \mathrm{E} 5$ \\
\hline
\end{tabular}

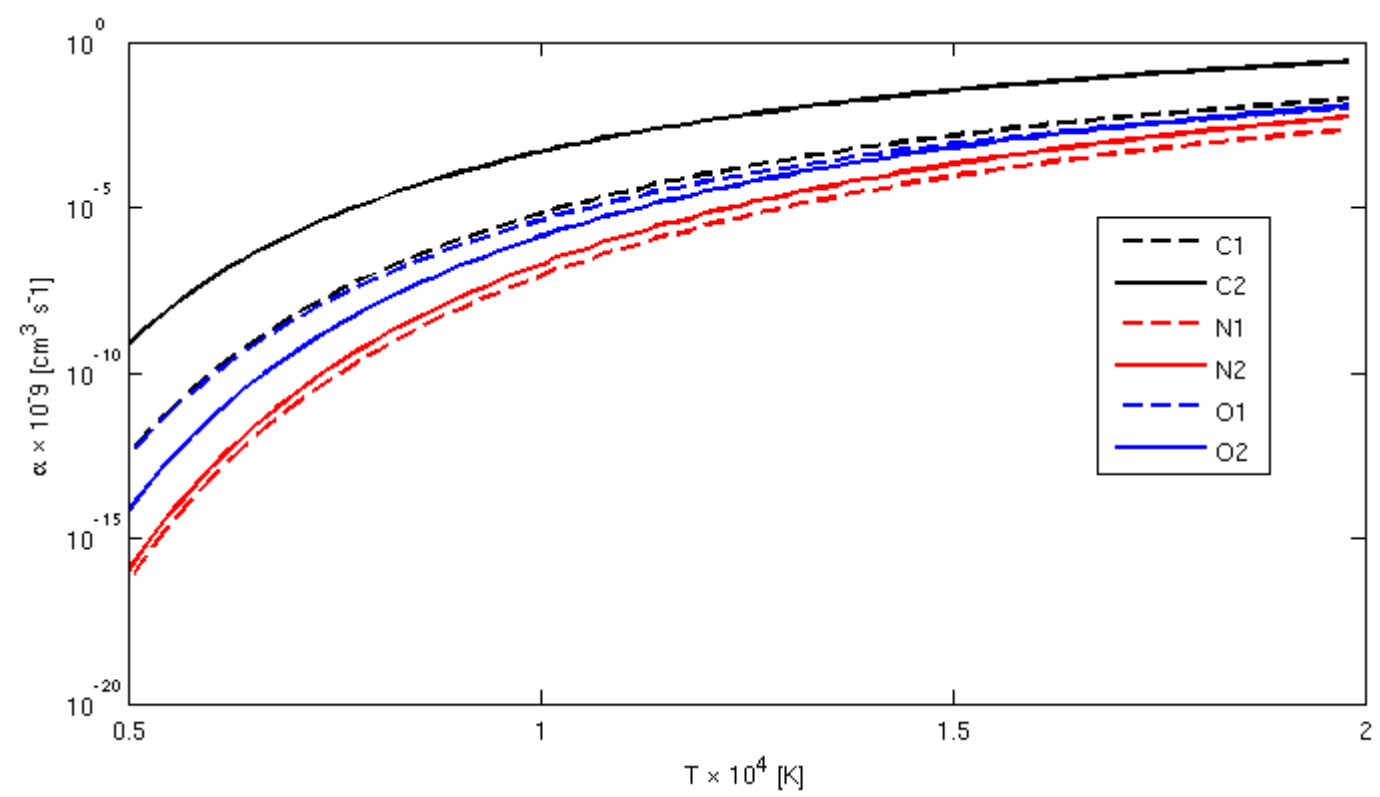

Figure 2. Dielectronic recombination coefficients of $C, N$ and $O$ atoms and ions using fitted data taken from [31] and using Eqn.18. 
In general, the low-temperature dielectronic recombination contribution is important at temperatures of about $1000-3000$ $\mathrm{K}$ and the high-temperature term is dominant above $20000 \mathrm{~K}$. Single electron recombination dominates at very low temperatures and is usually an important contributor in the region 5000 - $20000 \mathrm{~K}$ [12].

The dielectronic recombination coefficients of $\mathrm{C}, \mathrm{N}$, and $\mathrm{O}$ using the fitted data are shown in Figure 2. In all cases, the dielectronic recombination coefficients depend on temperatures. The results have shown that for carbon ion (C2), the dielectronic recombination coefficient shifts more upwards than the other elements. The dielectronic recombination coefficients other elements like $\mathrm{N}$ and $\mathrm{O}$ depends on temperature with a slow rate. The dielectronic recombination coefficients are bound states of the recombined system that are shown in Figure 2 significantly populated by the cascading the processes from the upper to the lower state.

\section{The Model Parameter}

We design this model using version c10.00 of the photoionization code cloudy [8] to compute the recombination coefficients. In this work, we assume the spherically symmetric, dust-free nebulae, and uniform density of hydrogen atoms plus ions $\left(\mathrm{n}=10^{2} \mathrm{~cm}^{-3}\right)$, the filling factor is 0.25 and helium abundance is constant throughout this model at a value of $(\mathrm{He} / \mathrm{H}=0.08)$. The photoionization calculation is stopped when the temperature falls below $100 \mathrm{~K}$. The relative abundances of heavy elements relative to hydrogen as the logarithm of numbers are given in Table 1 . We also assumed the central star had an ionized photon luminosity Q (Ho), Q

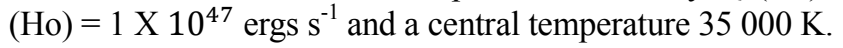

Table 2. Elemental abundances values Relative to hydrogen $(\log (X / H)$.

\begin{tabular}{ll}
\hline Element & Abundances \\
\hline Helium & -1.0 \\
Carbon & -3.45 \\
Nitrogen & -4.17 \\
Oxygen & -3.25 \\
Neon & -4.15 \\
Sulphur & -4.75 \\
\hline
\end{tabular}

\section{Results and Discussion}

There are four different temperatures selected to compute the radial, dielectronic and total recombination coefficients which are tabulated in Tables 3,4 and 5. The dielectronic recombination of the $\alpha_{d i}\left(X^{i}\right)$ are computed with the general expression given by [2]. For each ion, $X^{i+1}$ the calculation of $\alpha_{d i}\left(X^{i}\right)$ involves a summation overall permitted transitions from the ground state. The dielectronic recombination result is shown in Table 3. The first column in the table shows the elements and ions. The second column consists of the new model and the pervious results calculated by [32] hereafter G88. In most of the ions, the new results and G88 do not agree with each other at the temperature of $5000 \mathrm{~K}$. The dielectronic recombination coefficients in the other three temperatures shown in third, fourth and fifth column shown in Table 3 are significant with previous results of G88. Lowtemperature dielectronic recombination coefficients have been given by [2] for ions of $\mathrm{C}, \mathrm{N}, \mathrm{O}, \mathrm{Ne}$, and $\mathrm{S}$ are comparable to the new one.

Table 3. Dielectronic recombination coefficients.

\begin{tabular}{|c|c|c|c|c|c|c|c|c|}
\hline \multicolumn{9}{|c|}{$\alpha_{d i}\left(10^{-13}\right) \mathrm{cm}^{-3} s^{-1}$} \\
\hline \multirow{2}{*}{ Ion } & \multicolumn{2}{|c|}{$\mathbf{T}=5000 \mathrm{~K}$} & \multicolumn{2}{|c|}{$T=10000 K$} & \multicolumn{2}{|c|}{$T=15000 \mathrm{~K}$} & \multicolumn{2}{|c|}{$T=20000 \mathrm{~K}$} \\
\hline & New & G88 & New & G88 & New & G88 & New & G88 \\
\hline C I & 2.65 & 5.2 & 3.77 & 2.9 & 4.77 & 2.2 & 9.86 & 1.8 \\
\hline C II & 64.2 & 87.9 & 43.3 & 60.6 & 38.1 & 47.9 & 44.2 & 41.0 \\
\hline C III & 358.0 & 233.8 & 196.1 & 131.1 & 167.0 & 91.9 & 214.0 & 71.8 \\
\hline N I & 7.86 & 8.5 & 4.67 & 5.2 & 3.36 & 3.6 & 3.99 & 2.7 \\
\hline N II & 6.97 & 13.4 & 6.52 & 20.4 & 6.36 & 21.4 & 10.8 & 21.0 \\
\hline N IV & 169.0 & 151.7 & 205.0 & 153.8 & 198.0 & 137.2 & 219.0 & 120.9 \\
\hline O I & 0.75 & 0.7 & 0.763 & 0.8 & 0.689 & 0.8 & 1.04 & 0.8 \\
\hline O II & 16.5 & 24.2 & 9.45 & 16.6 & 7.47 & 12.9 & 6.71 & 10.7 \\
\hline O III & 60.0 & 78.1 & 78.5 & 113.9 & 69.7 & 110.1 & 59.3 & 100.7 \\
\hline O IV & 60.1 & 414.8 & 426.0 & 343.5 & 358.0 & 302.6 & 318.0 & 288.3 \\
\hline $\mathrm{Ne} \mathrm{I}$ & 0.564 & & 0.209 & & 0.116 & & 7.65 & \\
\hline $\mathrm{Ne}$ II & 5.71 & & 4.05 & & 3.10 & & 2.44 & \\
\hline S I & 1.97 & & 3.78 & & 5.91 & & 8.88 & \\
\hline S II & 18.5 & & 17.0 & & 19.0 & & 22.3 & \\
\hline S III & 126.0 & & 81.40 & & 66.0 & & 62.1 & \\
\hline S IV & 819.0 & & 160.0 & & 145.0 & & 139.0 & \\
\hline
\end{tabular}

The radiative recombination coefficients of ions are described by the following reaction.

$$
X^{i+1}+e \rightarrow X^{i-1}+h v
$$

The results which are produced by the interaction of the ion with electron are shown in Table 4. The first column stands for element and ions and the rest columns 2 to 5 shows the data of the new results and previous calculated by G88 
and J73 at different four temperatures. The result we obtained in the new model and the pervious G88 and J73 are insignificant to each other. In column 3 we have three data. The new data are calculated using photoionization codes almost comparable to each of the pervious data. Moreover, we have compared with the work that was done by [33], for both dielectronic and radiative Recombination are shown in Table 4 and 5 from excited levels within the ground state is different (smaller) compared to that for the ground level, due to the presence of additional autoionization pathways.

Table 4. Radiative recombination coefficients $\alpha_{R}\left(10^{-13}\right) \mathrm{cm}^{-3} \mathrm{~s}^{-1}$.

\begin{tabular}{llllllllll}
\hline \multirow{2}{*}{ Ions } & \multicolumn{2}{c}{ T=5000 K } & \multicolumn{2}{c}{ T=10 000 K } & \multicolumn{3}{c}{ T=15 000 K } & \multicolumn{2}{c}{ T=20 000 K } \\
\cline { 2 - 10 } & New & G88 & New & $\mathbf{G 8 8}$ & $\mathbf{J 7 3}$ & New & $\mathbf{G 8 8}$ & New & $\mathbf{G 8 8}$ \\
\hline C I & 7.93 & 7.4 & 4.72 & 4.66 & 4.7 & 3.72 & 3.5 & 3.23 & 2.29 \\
C II & 39 & 37.8 & 24.9 & 2.45 & 2.3 & 19.1 & 19 & 15.8 & 15.8 \\
C III & 81.9 & 78.4 & 51.4 & 5.05 & 3.2 & 38.9 & 38.9 & 31.9 & 32.2 \\
C IV & 140 & 132 & 86.7 & 84.5 & 75 & 64.9 & 64.8 & 52.7 & 53.5 \\
N I & 5.99 & 6.4 & 3.76 & 3.92 & 4.1 & 2.89 & 0.9 & 2.45 & 2.4 \\
N II & 37.4 & 35.5 & 24 & 22.8 & 22 & 18.4 & 17.6 & 15.2 & 14.6 \\
N III & 90.4 & 84.1 & 57.7 & 54.4 & 50 & 44.2 & 42 & 36.5 & 34.9 \\
N IV & 153 & 148 & 96.3 & 95.5 & & 73.1 & 73.7 & 60 & 61.1 \\
O I & 4.48 & 5.5 & 2.72 & 3.31 & 3.1 & 2.03 & 2.4 & 1.67 & 1.9 \\
O II & 33.4 & 32.3 & 21.3 & 20.5 & 20 & 16.3 & 15.6 & 13.5 & 12.9 \\
O III & 89.7 & 84.1 & 57.5 & 54.3 & 51 & 44.1 & 41.9 & 36.5 & 34.8 \\
O IV & 165 & 159 & 106 & 103 & 96 & 81.2 & 79.7 & 67.2 & 66.3 \\
Ne I & 3.38 & 4.9 & 1.92 & 0.82 & 2.2 & 1.38 & 2 & 1.08 & 1.6 \\
Ne II & 24.7 & 27.6 & 15.2 & 17.1 & 15 & 11.5 & 12.8 & 9.35 & 10.4 \\
Ne III & 75 & 69.7 & 47.5 & 44.4 & 44 & 36.3 & 33.9 & 29.9 & 28 \\
Ne IV & 159 & 152 & 102 & 98.1 & 91 & 78.1 & 75.7 & 64.6 & 62.8 \\
S I & 6.35 & 7.1 & 4.1 & 4.65 & 4.1 & 3.18 & 3.3 & 2.65 & 2.7 \\
S II & 29 & 37.9 & 18 & 24.2 & 18 & 13.6 & 18.6 & 11.2 & 15.4 \\
S III & 45.3 & 57.7 & 27 & 35.3 & 27 & 20 & 26.3 & 16.1 & 21.2 \\
S IV & 123 & 119 & 77 & 74 & 40.1 & 58.3 & 55.7 & 47.7 & 45.4 \\
\hline
\end{tabular}

Table 5. Total recombination coefficients $\left(\alpha_{T}\left(10^{-10}\right) \mathrm{cm}^{-3} \mathrm{~S}^{-1}\right)$.

\begin{tabular}{lllll}
\hline Ions & $\mathbf{T}=\mathbf{5 0 0 0} \mathbf{K}$ & $\mathbf{T}=\mathbf{1 0} \mathbf{0 0 0} \mathbf{K}$ & $\mathbf{T}=\mathbf{1 5} \mathbf{0 0 0} \mathbf{K}$ & $\mathbf{T}=\mathbf{2 0} \mathbf{0 0 0} \mathbf{~ K}$ \\
\hline C I & 1.12 & 0.956 & 0.962 & 1.49 \\
C II & 11.5 & 7.68 & 6.48 & 6.83 \\
C III & 49.2 & 27.8 & 23.3 & 28 \\
C IV & 15.7 & 9.76 & 7.35 & 6 \\
N I. & 1.55 & 0.949 & 0.708 & 0.733 \\
N II & 4.96 & 3.43 & 2.8 & 2.96 \\
N III & 43 & 31.8 & 25.3 & 211.4 \\
N IV & 35.9 & 33.9 & 30.7 & 31.8 \\
O I & 0.585 & 0.392 & 0.308 & 0.308 \\
O II & 5.57 & 3.46 & 2.69 & 2.29 \\
O III & 16.7 & 15.3 & 12.9 & 10.9 \\
O IV & 85.5 & 59.9 & 49.8 & 43.8 \\
Ne I & 0.44 & 0.24 & 0.169 & 0.132 \\
Ne II & 3.39 & 2.17 & 1.65 & 1.34 \\
Ne III & 40.9 & 20.5 & 14.8 & 12 \\
Ne IV & 55.2 & 34.5 & 26 & 21.5 \\
S I & 928 & 0.887 & 1.03 & 1.31 \\
S II & 5.3 & 3.94 & 3.7 & 3.82 \\
S III & 19.1 & 12.2 & 9.74 & 14.5 \\
S IV & 105 & 99.9 & 89.5 & 80.3 \\
\hline
\end{tabular}

Total recombination coefficients with the density of each element at the different stages of ionization are presented in Table 5. This gives the total recombination coefficients which are greater than both radiative and dielectronic recombination coefficients and have value more than $10^{-10} \mathrm{~cm}^{-3} \mathrm{~s}^{-1}$. In particular elements like, $\mathrm{O}, \mathrm{Ne}$ and $\mathrm{S}$ elements at the ground state, the total recombination coefficients are smaller than the rest stages. This is may be due to their low relative abundances of elements and the recombination cross sections of ions are relatively small than [34]. The present total recombination rate coefficients for oxygen ions $\mathrm{O}$ I to $\mathrm{O}$ IV agree very well with the earlier work done by [12].

\section{Conclusions}

We have used code cloudy (version c10.00) to design the model and to generate data for total, radiative and dielectronic recombination coefficients of $\mathrm{C}, \mathrm{N}, \mathrm{O}, \mathrm{Ne}$, and $\mathrm{S}$ at four different selected temperatures $5000,10000,15000$ and $20000 \mathrm{~K}$. The results are shown in Tables 3, 4 and 5. From our analysis, we come to these conclusions: from the CLOUDY photoionization model we obtain for a limited space of physical parameter radiative, dielectronic and total recombination coefficients for each ion, which agree for some of them with the results of [32]. But the result we obtained for dielectronic recombination coefficients shown in Table 4 has a slight difference with the results obtained by [32]. We have concluded that the recombination coefficients depend on the effective temperature and not depend on abundances of elements. The calculation can be improved at high effective temperature for dielectronic recombination coefficients of elements. The other main point we obtained from this work, the die-electronic recombination coefficients for low temperature which have significant impacts on the electron-ion recombination. But this value is much smaller than the radiative recombination.

\section{References}

[1] P'equignot D., Petitjean P., \&Boission C., 1991, A\&A, 251, 680.

[2] Burgess A., 1965, ApJ, 17, 198.

[3] Tarter C. B., 1973, ApJ, 168, 313.

[4] Tarter C. B., 1971, ApJ, 168, 313.

[5] Aldrovandi S. M. V., and Pequigno D., 1973, A\&A, 25, 137.

[6] Aldrovandi S. M. V., and Pequigno D., 1973, Rev. Bras. Fis. $4,491$.

[7] Gould R. J., 1978, ApJ, 219, 250.

[8] Ferland G. J., 2011, Hazy a brief introduction to cloudy, University of Kentucky internal report.

[9] Osterbrock D. E., 1989, Astrophysics of Gaseous Nebulae and Active Galactic Nuclei, University of Science Book, California, Santa Crux.

[10] Susmita ChakravortyAjit K. Kembhavi, Martin Elvis, Gary Ferland and N. R. Badnell, 2008, MNRAS, 384, 24.

[11] Burgess A. and Seaton, M. J., 1960a, MNRAS, 120, 76.

[12] Sultana N., 1999, ApJS, 120, 131. 
[13] Osterbrock D. E., 1974, Astrophysics of Gaseous Nebulae, San Francisco: Freeman.

[14] Woods D. T., Shull J. M., \& Sarazin C. L., 1981, ApJ, 399, 401.

[15] Michael A. Dopita\& Ralph S. Sutherland, 1999, Diffuse matter in the universe. The Australian National University, Can-berra.

[16] Anil K. Pradhan and Sultana N. Nahar, 2011, Atomic Astrophysics and Spectroscopy, United Kingdom at the University Press, Cambridge.

[17] Dopita M. A. and Sutherland R. S., 2001, Diffuse Matter in the Universe, Subtitle, Edition, Physics of Monograph.

[18] Shull J. M. \& Steenberg, M. V., 1982, ApJ, 48, 95-107.

[19] Liu X., 2010, arxiv: 1001.3715v2 [astro.ph.GA]. The dark secrets of gaseous nebulae highlights from deep spectroscopy.

[20] Jon C. Weisheit, ApJ, 1973, 185, 877.

[21] Bates D. R. and Massey H. S. W., 1943. The negative ions of atomic and molecular oxygen. Philosophical Transactions and of the Royal Society of London, Series A, 239(806): 269-304.

[22] Massey H. S. W. and Bates D. R., 1942. The properties of neutral and ionized atomic oxygen influence on the upper atmosphere. Reports in Physics, 9(1), 62-74.

[23] Orban Z., Altun, A., Ka'llberg A., Simonsson, G., Andler A.,
Pa al M., Blom P., La'fgren S., Trotsenko S., BAuhm \& Schuch R., 2009 A \& A 498, 909.

[24] Burgess A., 1964. Dielectronic recombination and the temperature of the solar corona. ApJ, 139, 776

[25] Sultana N. Nahar and Anil K. Pradhan, 2006, ApJS, 162, 417.

[26] Fogel M., Bandell N. R., Glans P., Loch S. D., Madzunkov S., Abdel-NabySh. A., Pindzola M. S., \& Schuch R., 2005, A\&A, 442,757

[27] Altun Z., Yumak A., Bandell N. R., Loch S. D., Pindzola M. S., 2006, A\&, 447, 1165.

[28] Drake G. W. F., 1996, in Drake G. W., ed., Atomic, Molecular, and Optical Physics Handbook. American Institute of Physics, Woodbury, New York, p. 154.

[29] Nussbaumer H. \& Storey P. J., 1983, A\&A, 126, 74.

[30] Sultana N. Nahar\& Anil K. Pradhan, 1995, ApJ, 447, 966

[31] Jacobs V. L., Davis J., Kepple P. C., and Blaha M., 1977b, Apj, 215, 690.

[32] Goharji A. A., 1988, Durham Thesis, Durham University

[33] Bandell N. R., 2005, A\&A, 447, 389.

[34] Williams R. E., Jenkins E. B., Baldwin J. A., Zhang Y., Sharpee B., Lellegrini E., and Philips M., 2008, ApJ, 677, 1100-1119. 